\title{
Identification of SARS-CoV-2 3CL Protease Inhibitors by a Quantitative High-throughput Screening
}

Wei Zhu, Miao Xu, Catherine Z. Chen, Hui Guo, Min Shen, Xin Hu, Paul Shinn, Carleen KlumppThomas, Samuel G. Michael, Wei Zheng*

National Center for Advancing Translational Sciences, National Institutes of Health, Rockville, MD 20850, USA

\section{Supplementary Information}

\section{Table of Contents}

- Figure S-1 Triage strategy used to screen compounds (pg. S-2)

- Figure S-2. Standard curve for counter screen (pg. S-3)

- Figure S-3. Concentration-response curves of identified compounds by $3 \mathrm{CL}^{\text {pro }}$ assay (pg. S-4)

- Figure S-4. Concentration-response curves of compounds showed apparent quenching effect in counter screen. (pg. S-5) 


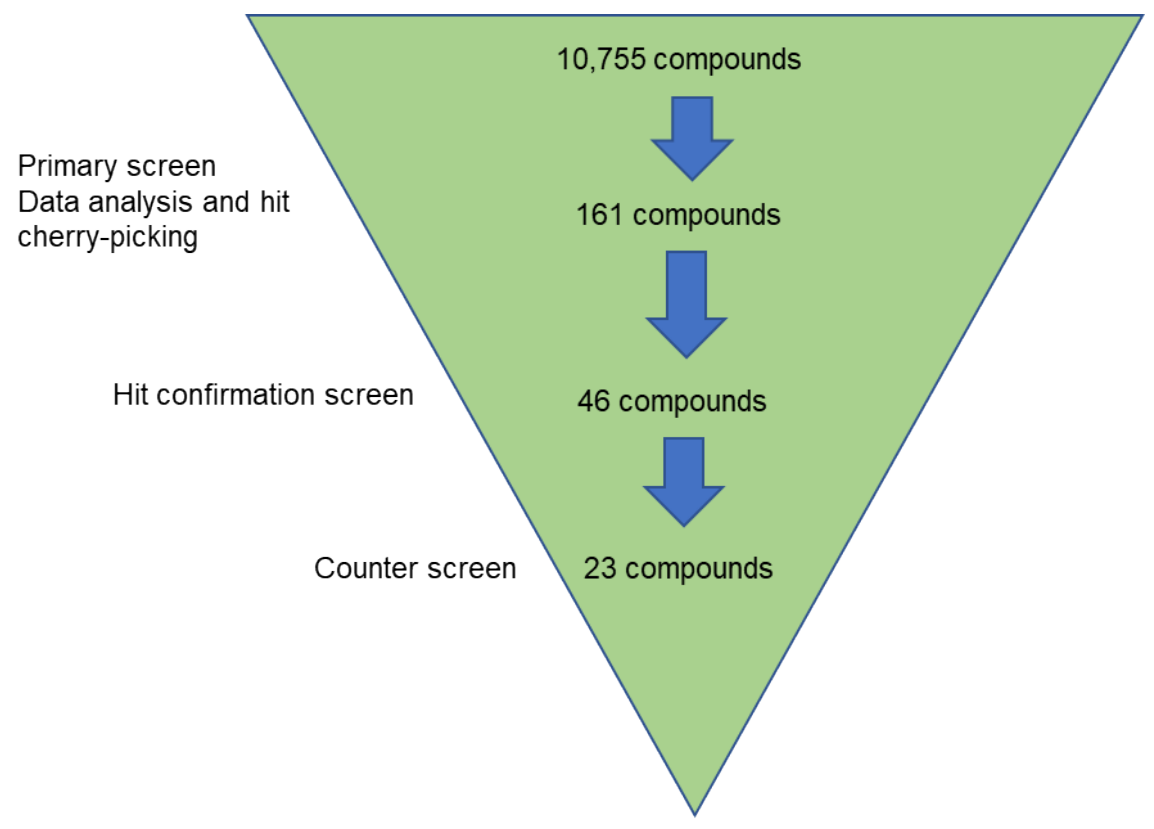

Figure S-1. Triage strategy used to narrow down the starting 10,755 small molecules from the primary HTS campaign which led to the identification of 23 compounds having $\mathrm{IC}_{50} \mathrm{~s}<30 \mu \mathrm{M}$, maximal inhibition $>60 \%$ against SARS-CoV-2 3CL ${ }^{\text {pro }}$. 


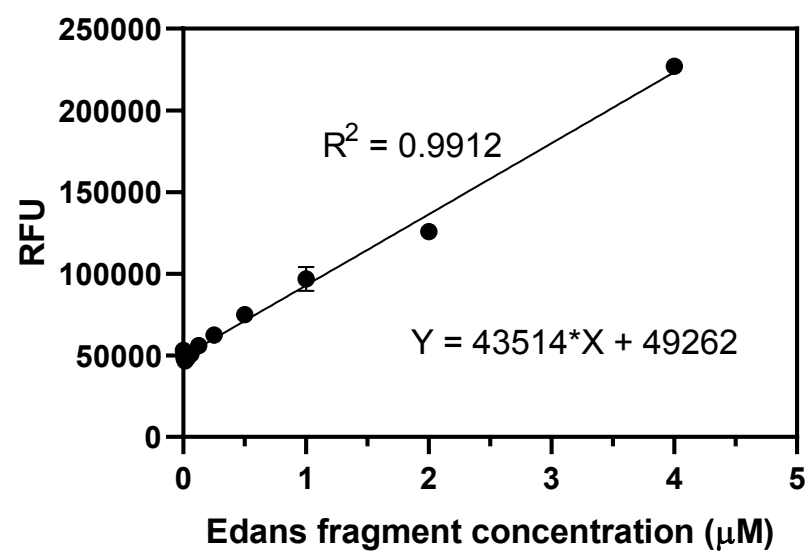

Figure S-2. Standard curve generated by diluting SGFRKME-Edans (fluorescent fragment in assay byproducts) in $20 \mu \mathrm{M}$ substrate solution. The relative fluorescent unit (RFU) is linearly proportional to the amount of SGFRKME-Edans. 

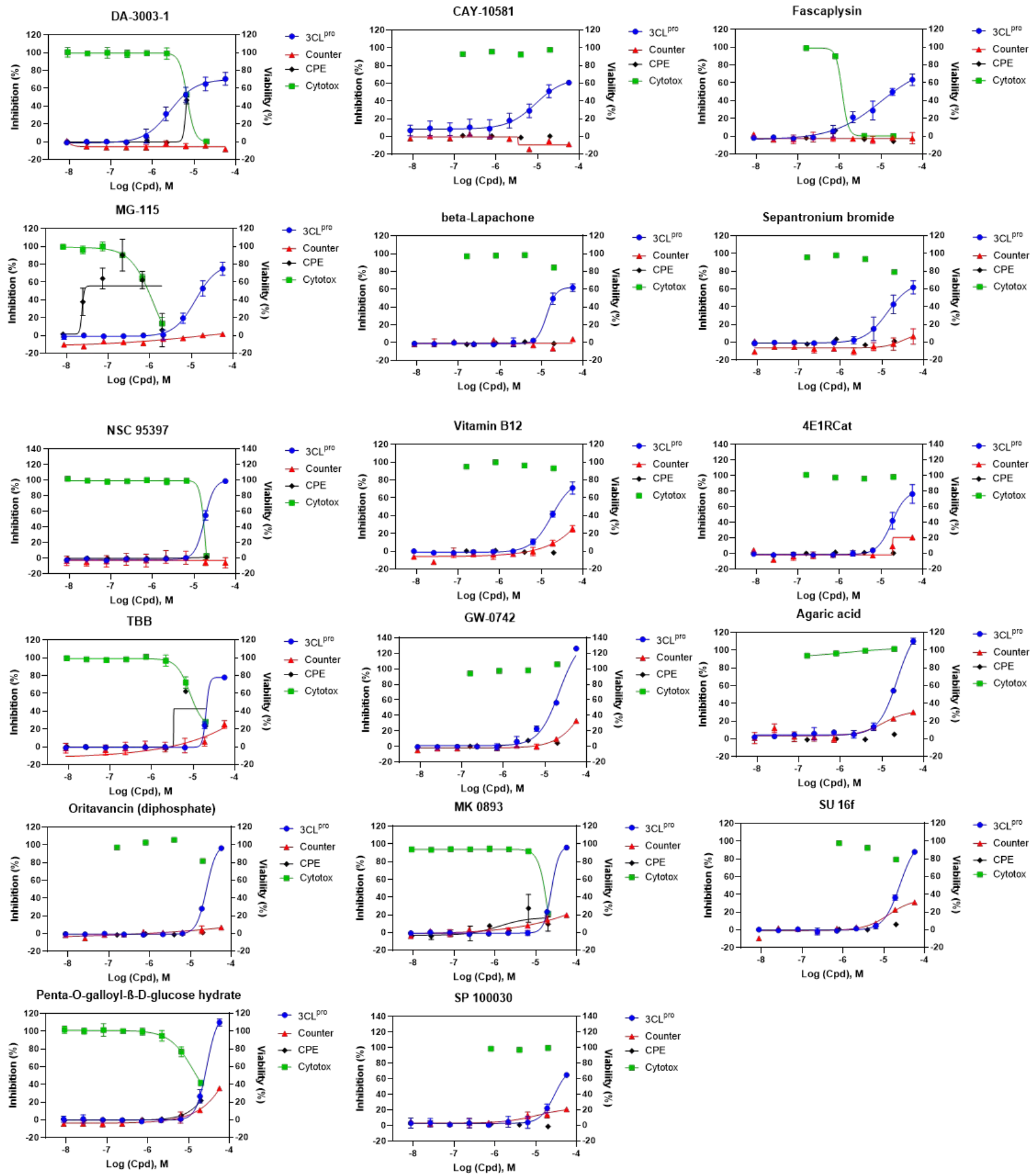

Figure S-3. Concentration-response curves of identified compounds by $3 \mathrm{CL}^{\text {pro }}$ assay. These compounds showed relatively negligible quenching effect relative to enzyme inhibitory activity. 

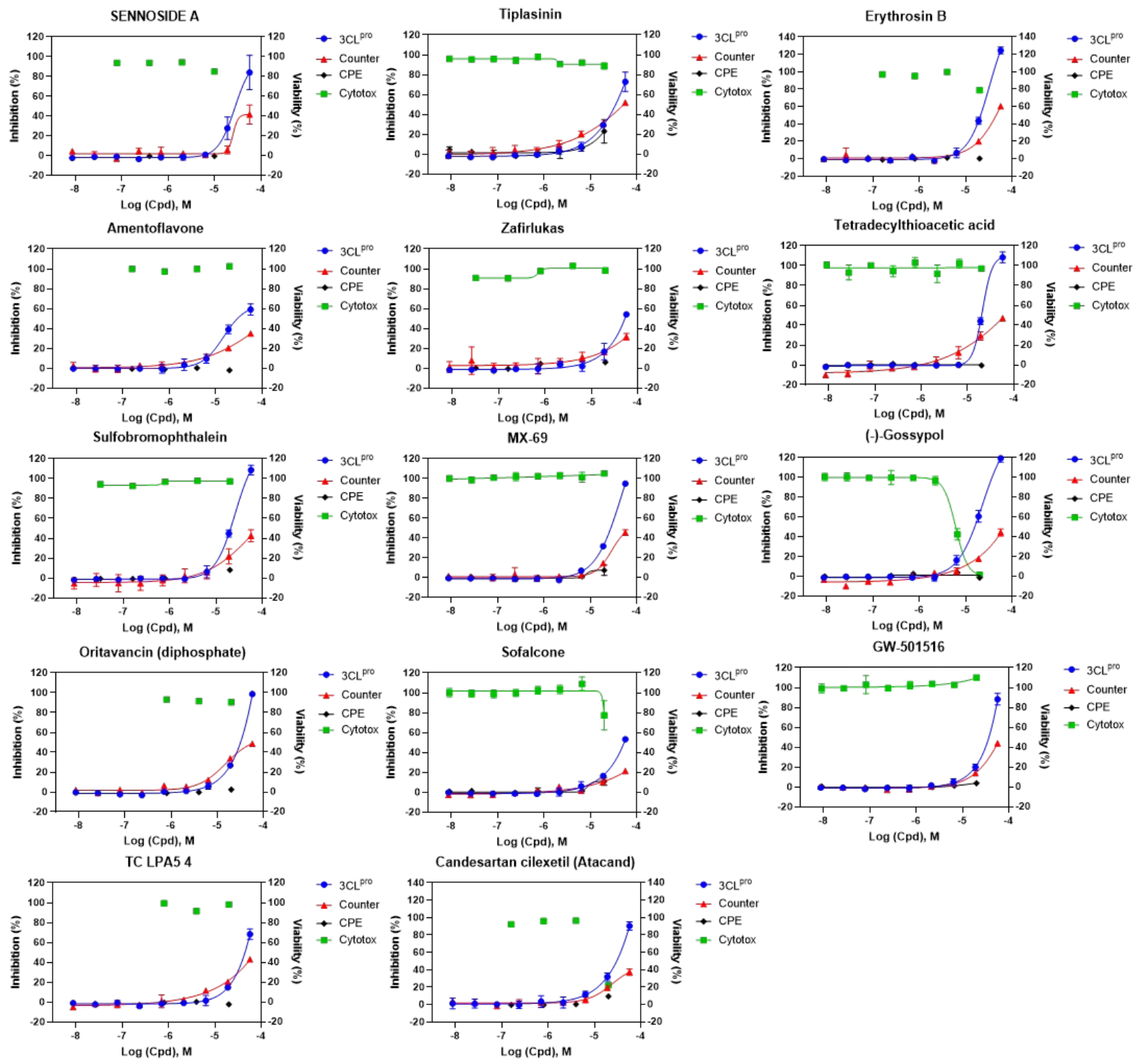

Figure S-4. Concentration-response curves of compounds showed apparent quenching effect in counter screen. 